\title{
Thrombomodulin Expression in Bladder Cancer Tissue and Its Association with Prognosis and Patient Survival
}

This article was published in the following Dove Press journal: Research and Reports in Urology

\author{
Joanne Watt (D) \\ Daniel G Maguire \\ Cherith N Reid \\ John V Lamont \\ Stephen P Fitzgerald \\ Mark W Ruddock (D)
}

Randox Laboratories Ltd, Molecular Biology, Crumlin, County Antrim BT29

4QY, Northern Ireland, UK
Correspondence: Mark W Ruddock Randox Laboratories Ltd, Molecular Biology, 55 Diamond Road, Crumlin, County Antrim BT29 4QY, Northern Ireland, United Kingdom

Tel +44289442 2413

Fax +44289445 2912

Email Mark.Ruddock@randox.com
Background: Decreased expression of thrombomodulin (TM) in bladder cancer tissue has been shown to be associated with cell proliferation, increased malignancy and a poor prognosis. The aim of this study was to investigate the immunoexpression of TM in bladder tissue cores by immunohistochemistry (IHC) and the relationship between TM score and patient survival for the following pathologies: transitional cell papillary carcinoma (TCPC), transitional cell carcinoma (non-papillary) (TCC), squamous cell carcinoma (SCC), adenocarcinoma, and sarcoma. TM immunoexpression was also evaluated in normal adjacent bladder tissue cores.

Methods: TM immunoexpression was assessed in $n=185$ formalin-fixed paraffin-embedded (FFPE) bladder tissue cores from $n=98$ patients by IHC. Tissue cores included TCPC $(n=29)$, TCC $(n=85)$, SCC $(n=21)$, adenocarcinoma $(n=12)$, sarcoma $(n=4)$, and normal tissue cores $(\mathrm{n}=34)$.

Results: TM immunoexpression scores are stronger in TCPC, TCC and SCC bladder cancer tissue cores with respect to adenocarcinoma and sarcoma (mean TM immunoexpression scores: 3.04, 2.57, 2.55, 1.55 and 1.19, respectively) (Kruskal-Wallis p<0.001). TM immunoexpression scores significantly decreased in bladder cancer tissue cores across both stage $(\mathrm{p}<0.001)$ and grade $(\mathrm{p}<0.001)$ (Kruskal-Wallis). Survival data were available for $\mathrm{n}=45$ bladder cancer patients (mean follow-up of 34 months). Applying a TM immunoexpression cut-off score of 3.0 demonstrated that patients with bladder cancer who had a TM immunoexpression score $<3.0$ had lower survival rates (median survival 23.5 months). In contrast, patients with TM immunoexpression scores $\geq 3.0$ had longer survival rates (median survival 40 months) (log-rank; $\mathrm{p}=0.045$ ).

Conclusion: TM immunoexpression in bladder cancer tissue may be a clinically relevant predictor of tumor progression and survival. Low expression of TM in bladder cancer biopsies or in recurrent bladder cancer may be indicative of a poor prognosis. TM immunoexpression could be used to guide clinical decision making.

Keywords: transitional urothelial carcinoma, tissue microarrays, immunohistochemistry, bladder cancer

\section{Introduction}

Bladder cancer is the 10th most common cancer in the UK, with over 10,000 new cases diagnosed in 2015 (male:female ratio 2.7:1.0). Bladder cancer is normally detected early with over $70 \%$ of patients diagnosed either stage I or II. However, over $17 \%$ of patients with bladder cancer present with metastatic disease. As such, the 10 -year patient survival rates for bladder cancer remain at $50 \%$ and accounted for over 5000 deaths in the UK in $2014 .^{1}$ 
Diagnosis of bladder cancer is determined by histopathological examination of tissues obtained from transurethral resection of the bladder tumor (TURBT)., ${ }^{2,3}$ Transitional cell carcinoma of the urinary bladder represents the most common histological type, accounting for almost $90 \%$ of all urothelial carcinomas. ${ }^{4}$ Transitional cell carcinomas represent two distinct diseases; transitional cell papillary carcinoma (TCPC), which are usually low-grade and non-invasive (superficial) and transitional cell nonpapillary carcinoma (TCC), which are usually high-grade and invasive. ${ }^{5,6}$ Squamous cell carcinomas (SCC) represent 1.2 to $5 \%$, primary adenocarcinomas 0.5 to $2 \%$, and sarcomas $<1 \%$ of all bladder cancer diagnosis. ${ }^{4}$

Seventy-five to $85 \%$ of bladder cancer patients present with non-muscle invasive disease, and are usually treated by TURBT. ${ }^{3,7}$ However, bladder cancer recurrence rates are high (30 to $70 \%$ ), with 20 to $30 \%$ of patients showing a progression in both stage and grade. ${ }^{8}$ National Institute of Clinical Excellence guidelines recommend routine follow-up cystoscopy every 3 to 12 months, depending on the patient risk category. Follow-up cystoscopy is important as recurrence rates are high and there is a risk of under-staging the disease. ${ }^{3}$ As such, bladder cancer is one of the most expensive cancers for the health service to treat due to the cystoscopic, radiologic, and interventional procedures used to guide treatment and management decisions. ${ }^{3,9-13}$

Bladder cancer lacks clinically useful biomarkers for predicting disease stage and clinical outcome. ${ }^{14}$ Therefore, there is a clinical need for the identification of biomarkers with the ability to identify the true malignant potential of a tumor, or at least to stratify patient risk for bladder cancer. $^{15}$

This observational pilot study examined thrombomodulin (TM) immunoexpression in bladder cancer tissue cores; namely TCPC, TCC, SCC, adenocarcinoma, sarcoma and normal bladder tissue, to determine if any relationship exists between TM immunoexpression, tumor stage, grade, and patient survival. The practical purpose of this study was to investigate the clinical utility of TM as a potential bladder cancer biomarker for predicting prognosis and patient survival.

\section{Materials and Methods Study Population}

Ninety-eight patient samples were included in this observational pilot study. In total, $\mathrm{n}=185$ FFPE bladder tissue cores across $\mathrm{n}=6$ tissue microarrays (TMAs) were investigated, to include TCPC 29/185 (15.68\%), TCC 85/ 185 (45.95\%), SCC 21/185 (11.35\%), adenocarcinoma ( $\mathrm{n}=8$ mucinous) $12 / 185$ (6.49\%), sarcoma 4/185 (2.16\%) and normal tissue controls 34/185 (18.38\%). Human tissue cores used in this observational study are de-identified and publicly available. They are thus exempt from the requirement of the Institutional Review Board (IRB) approval (Exempt Category 4). The American Joint Committee on Cancer (AJCC) staging (v 7.0) was used for clinical stage I, II, III, IV and TNM. ${ }^{16}$

\section{Tissue Microarrays and Reagents}

Human bladder TMAs (T121a, T122, T123, T124, T125, HBlaU066Su01) were purchased from US Biomax, Rockville, MD 20849, US. Normal urinary bladder tissue sections (BLA01) were obtained from Pantomics, Inc 2600 Hilltop Drive, Suite C130, BLDG B, Richmond, CA 94806. Rabbit monoclonal anti-TM [EPR4051] antibody (AB108189), HistoReveal (AB103720) and DAB substrate kit (AB64238) were purchased from Abcam, Cambridge Science Park Milton Rd, Milton, Cambridge CB4 0FL, UK. Clearing reagent HistoClear (HS-200) was obtained from National Diagnostics, Atlanta, Georgia, US. Bovine serum albumin (BSA) (A4503), goat anti-rabbit HRP (A0545) conjugate, absolute ethanol $(24,103)$, Triton X-100 (T9284-500mL), 30\% hydrogen peroxide (95321), Eukitt ${ }^{\circledR}$ mounting medium (03989-100mL), and counterstains Harris hematoxylin (HHS32) and eosin (HT110116) were obtained from Sigma, Poole, UK. Phosphate buffered saline (10x) (AM9624) was obtained from Invitrogen by Thermo Fisher Scientific Baltics UAB, V. A. Graiciuno 8, Vilnius, LT-02241. All other reagents, unless otherwise indicated, were obtained from Sigma, Poole, UK.

\section{Immunohistochemistry}

This work has been described previously. ${ }^{17}$ Briefly, tissue sections were deparaffinized in Histoclear and then rehydrated. The slides were blocked at room temperature for 2 hours in $10 \%$ blocking reagent. Antigen retrieval was performed using HistoReveal (as per manufacturer's instructions). The TMAs were then incubated overnight at $4{ }^{\circ} \mathrm{C}$ with gentle rocking with a rabbit monoclonal antiTM antibody (1:750) prepared in 1x PBS ( $\mathrm{pH} 7.2) / 1 \%$ BSA. The negative control was 1x PBS ( $\mathrm{pH} 7.2) / 1 \%$ BSA. Following overnight incubation, the TMAs were washed for $3 \times 5$ minutes in $1 \times$ PBS (pH 7.2), Triton $\mathrm{X}-100(0.1 \%)$ (Wash Solution), incubated with hydrogen 
peroxide $(0.3 \%)$ (15 minutes), washed again with wash solution ( $3 \times 5$ minutes) and then incubated with a secondary antibody (goat anti-rabbit HRP 1:2000 prepared in 1x PBS (pH 7.2)/1\% BSA). Following 3 $x$ 5-minute washes, the TMAs were DAB stained for 10 minutes at room temperature, rinsed in tap water, and then counterstained with Harris hematoxylin (1 minute). The TMAs were then dehydrated through ethanol (50, 70, 90, 95 and $100 \%$ ) to Histoclear. Eukitt ${ }^{\mathbb{B}}$ mounting medium was added to the TMAs and a coverslip applied.

Immunoreactivity scoring for TM immunoexpression was undertaken by two observers (MWR and DGM) using the European Organization for Research and Treatment of Cancer-Gynecological Cancer Cooperative Group scoring system. ${ }^{18}$ Briefly, tissue sections that are negative (score 0 ), weakly positive (score 1), positive (score 2), strongly positive (score 3 ) and very strongly positive (score 4).

\section{Microscopy}

Micrograph images were captured by an Olympus BX43F series microscope using an Olympus SC50 CMOS color camera (Olympus, Tokyo, Japan) and cellSens Entry 1.15 imaging capture software.

\section{Statistical Analysis}

Statistical analyses were performed using R, Version 3.5. ${ }^{19}$ The Kruskal-Wallis test was performed to identify if there were differences between groups. Post-hoc, the Wilcoxon rank sum test was used to identify which groups were significantly different. Kaplan-Meier plots were used to evaluate bladder cancer tissue TM immunoexpression scores versus patient survival. Logistic regression was used to examine the relationship between age, gender and TM immunoexpression score.

\section{Results}

Patient clinicopathological characteristics are described in Table 1.

Representative micrographs for TM immunoexpression in bladder cancer tissue cores TCPC (grade II, stage NA, T1-M0), TCC (grade II, stage 1, T1N0M0), SCC (grade II, stage 2, T2N0M0), and adenocarcinoma (grade I, stage 2, T2N0M0) are described in Figures 1-4, respectively. Two micrographs for two TCC patients (aged 75 and 55 years, respectively) with grade III and stage 3 tumors with contrasting TM immunoexpression scores are described in Figure 5.
Table I Clinicopathological Characteristics

\begin{tabular}{|l|l|}
\hline Characteristics & Number of Patients (\%) \\
\hline Age (mean years \pm SD) & $63.2 \pm 13.4$ \\
Range (years) & $15-85$ \\
\hline Gender & \\
Male & $78(79.6 \%)$ \\
Female & $20(20.4 \%)$ \\
Male:female ratio & $3.9: 1$ \\
\hline Patient Groups & \\
Normal & $8(8 \%)$ \\
TCPC & $17(17 \%)$ \\
TCC & $57(58 \%)$ \\
SCC & $9(9 \%)$ \\
Adenocarcinoma & $5(5 \%)$ \\
Sarcoma & $2(2 \%)$ \\
\hline Histological Grade & \\
Grade 0 & $9(9 \%)$ \\
Grade I & $6(6 \%)$ \\
Grade I-II & $3(3 \%)$ \\
Grade II & $30(31 \%)$ \\
Grade II-III & $27(28 \%)$ \\
Grade III & $22(22 \%)$ \\
Ungraded & $1(1 \%)$ \\
\hline Pathological Stage & $11(11 \%)$ \\
Stage 0 (Ta, Tis) & $8(8 \%)$ \\
Stage I (TI) & $32(33 \%)$ \\
Stage II (T2a, T2b) & $27(28 \%)$ \\
Stage III (T3a, T3b, T4a) & $6(6 \%)$ \\
Stage IV (T4b/any N+/MI) & $14(14 \%)$ \\
Un-staged & \\
\hline
\end{tabular}

Abbreviations: TCPC, transitional cell papillary carcinoma; TCC, transitional cell non-papillary carcinoma; SCC, squamous cell carcinoma.

Tumor staging was available for $138 / 152$ (90.8\%) tissue cores from $\mathrm{n}=84$ patients however, one section was lost during antigen retrieval (TMA array T122 core B6, TCC) leaving 137/152 (90.1\%) tissue cores for analyses. Kruskal-Wallis analyses identified significant differences between TM immunoexpression scores within the groups $(\mathrm{p}<0.001)$ and the results with post hoc Wilcoxon rank sum analyses are described in Figure 6.

Information on tumor grade was available for 147/152 (96.7\%) tissue cores from $n=88$ patients. Kruskal-Wallis analyses identified significant differences between TM immunoexpression scores for each stage $(p<0.001)$ and the results with post hoc Wilcoxon rank sum analyses are described in Figure 7.

There was a negative relationship between patient histopathology and TM immunoexpression score. Kruskal- 


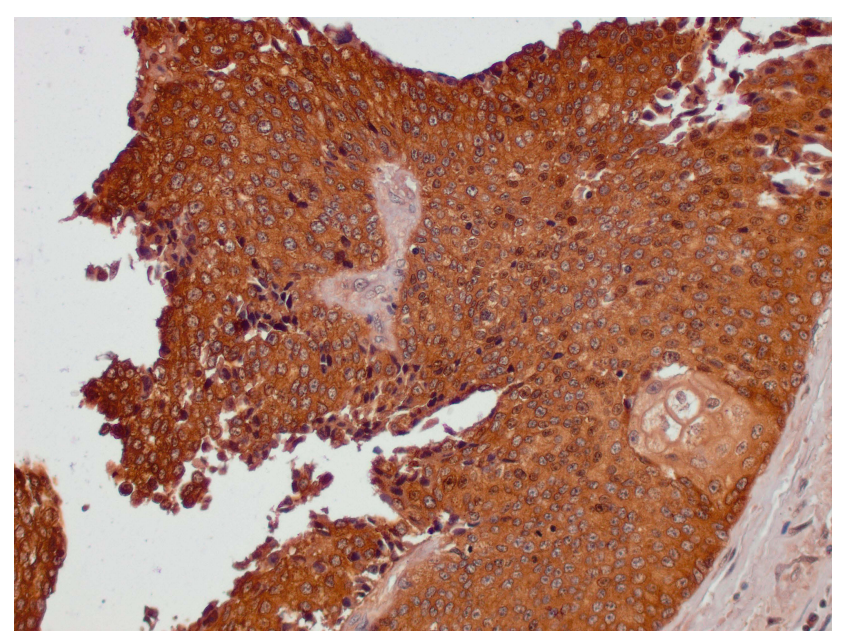

Figure I Bladder carcinoma, papillary (TCPC) (HBlaU066Su0I, position A8) (77year-old male, grade II, TI-MO, malignant bladder cancer) probed anti-TM, counterstained with hematoxylin (magnification 200x).

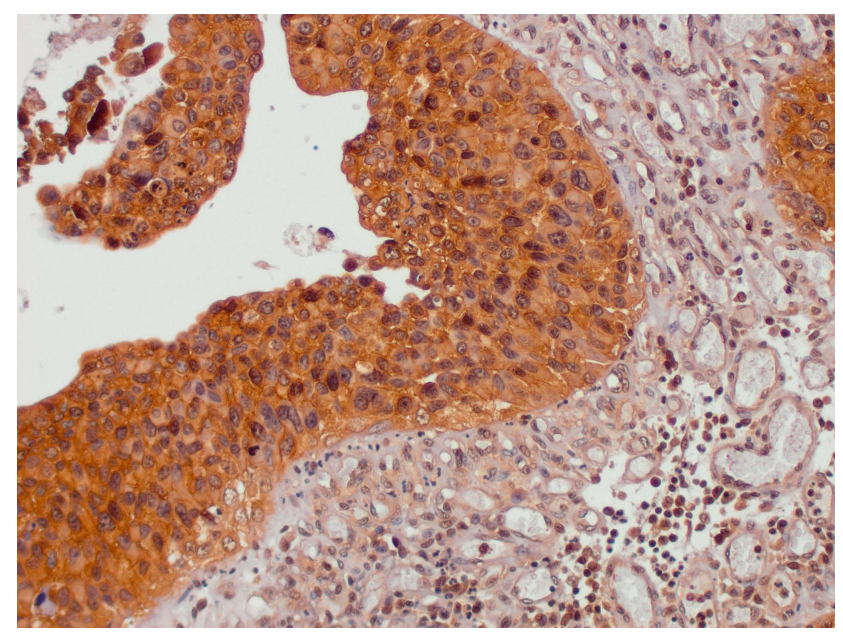

Figure 2 Bladder carcinoma (TCC) (HBlaU066Su0I, position Cl0) (74-year-old male, grade II, stage I, TINOM0, malignant bladder cancer) probed anti-TM, counterstained with hematoxylin (magnification 200x).

Wallis analyses identified significant differences between groups $(\mathrm{p}<0.001)$. The TM immunoexpression scores for normal, TCPC, TCC, SCC, adenocarcinoma and sarcoma tissue cores are described in Figure 8 with post hoc Wilcoxon rank sum analyses. The TM immunoexpression score was stronger for TCPC $>$ TCC $>$ SCC $>$ adenocarcinoma $>$ sarcoma.

Survival data were available for $\mathrm{n}=45$ bladder cancer patients (mean follow-up of 34 months). Median age was 73 years (range: $50-85$ years) with $82 \%$ males. Applying a TM immunoexpression cut-off score of 3.0 demonstrated that patients with bladder cancer who had a TM score $<3.0$ had lower survival rates (median survival 23.5 months). In contrast, patients with TM immunoexpression scores $\geq 3.0$

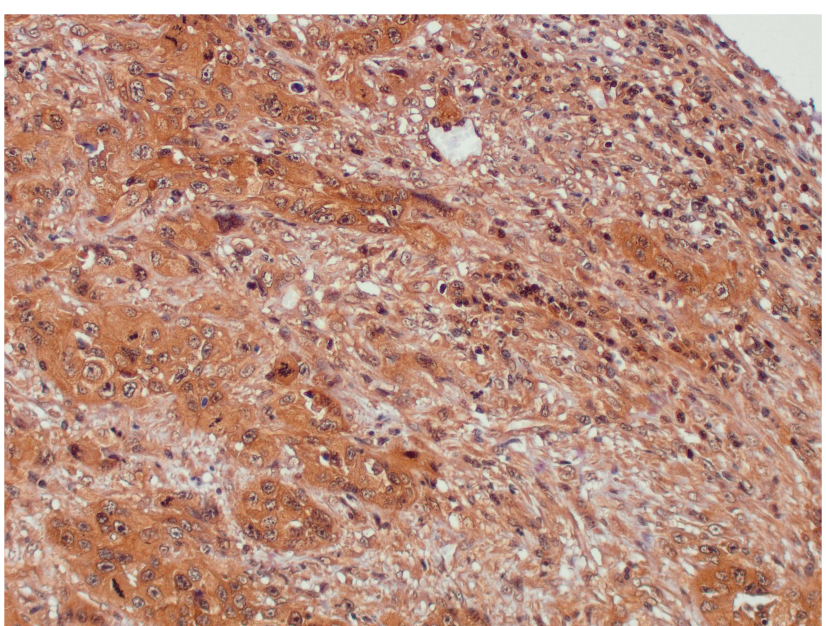

Figure 3 Bladder carcinoma, squamous carcinoma (SCC) (HBlaU066Su0I, position BI0) (68-year-old male, grade II, stage 2, T2NOM0, malignant bladder cancer) probed anti-TM, counterstained with hematoxylin (magnification 200x).

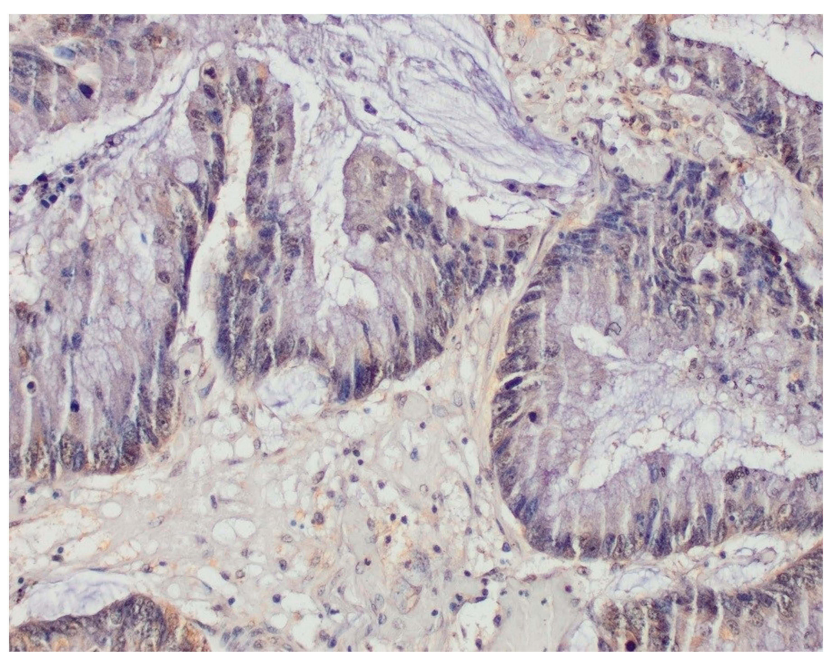

Figure 4 Bladder adenocarcinoma (mucinous) (TMA TI24, position B4) (48-yearold male, grade I, stage 2, T2NOM0, malignant bladder cancer) probed anti-TM, counterstained with hematoxylin (magnification 200x).

had significantly longer survival rates (median survival 40 months) (log-rank; $\mathrm{p}=0.045$ ) (Figure 9).

Logistic regression was used to examine the relationship between age, gender and TM immunoexpression score for TCPC (low risk of progression) versus all other cancers (TCC, SCC, adenocarcinoma and sarcoma; high risk of progression). However, only TM immunoexpression score was significant in the regression (sensitivity 71.2\%; specificity 76.5\%) (AUC 0.761).

\section{Discussion}

TM is well characterized as an anticoagulant in vascular endothelial cells. However, TM impacts other physiological 

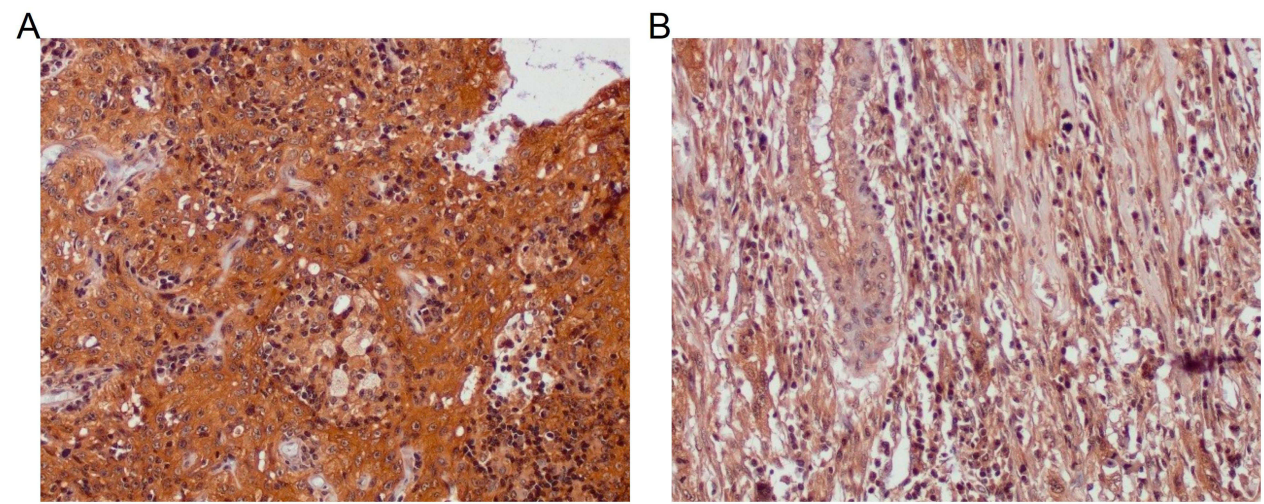

Figure 5 Bladder carcinoma TM immunoexpression between two independent patient tissue cores both graded III and staged 3. Left micrograph (A) HBlaU066Su0I TMA, position A2, 75-year-old male, grade III, stage 3, T3N0M0, malignant bladder cancer; right micrograph (B), HBlaU066Su0I TMA, position B9, 55-year-old male, grade III, stage 3, T3-M0, malignant bladder cancer. Tissue cores probed anti-TM and counterstained with hematoxylin (magnification 200x). The bladder cancer patient whose biopsy is described in the left micrograph (with evident TM immunoexpression (score $\geq 3.0$ )) is alive 82 months after surgery. However, the bladder cancer patient whose biopsy is described in the right micrograph (with low TM immunoexpression (TM score <3.0)) only survived 9 months following their surgery.

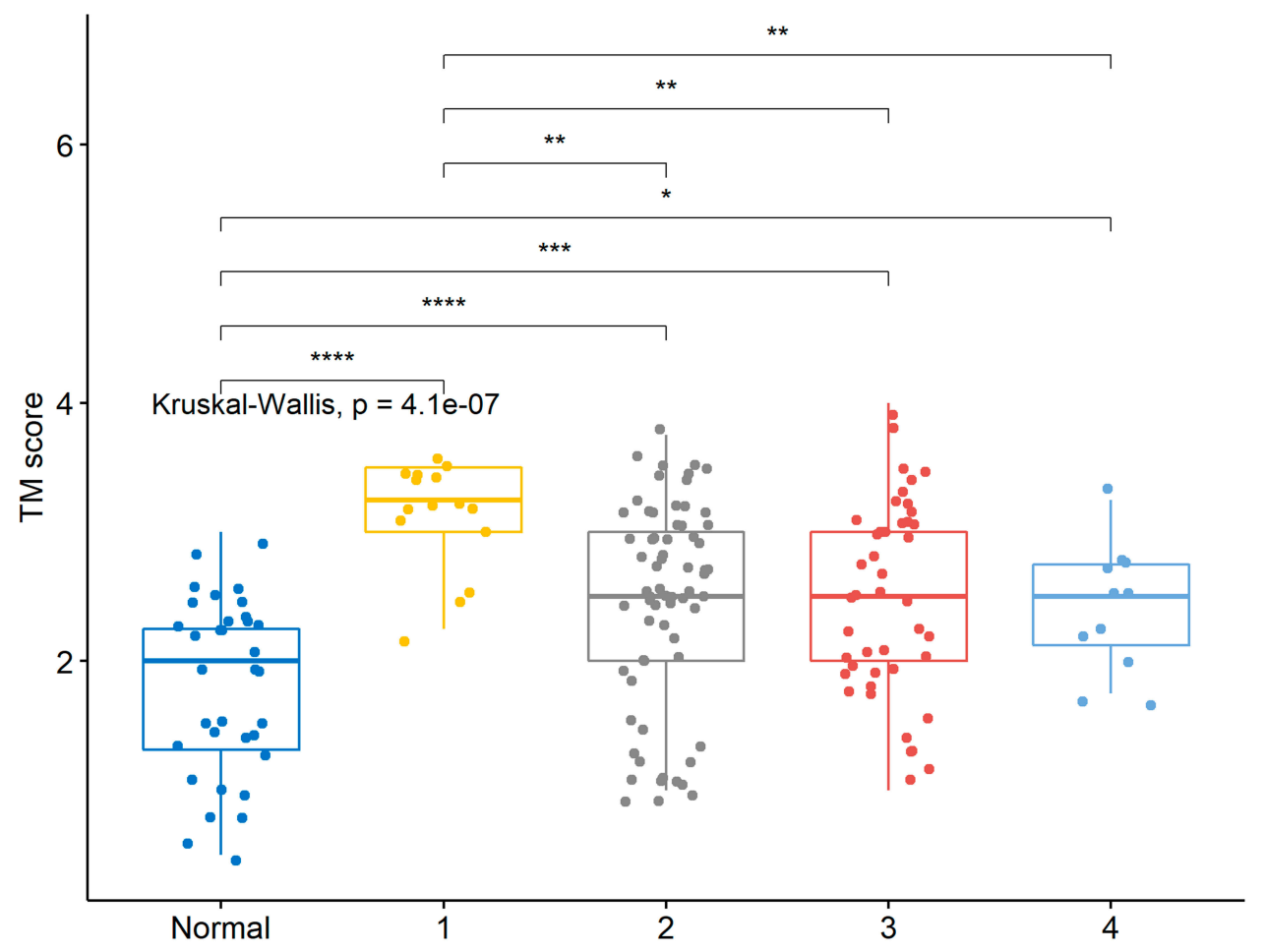

Figure 6 TM immunoexpression scores were significantly lower in normal bladder tissue. TM immunoexpression scores in bladder cancer tissue decreased across stage $\left({ }^{*} \mathrm{p}\right.$ $\left.\leq 0.05,{ }^{* *} \mathrm{p} \leq 0.01, * * * \mathrm{p} \leq 0.001, * * * * \mathrm{p} \leq 0.0001\right)$.

and pathophysiological processes including anti-proliferative and anti-metastatic effects in malignant neoplasms. ${ }^{20}$ As such, TM has been shown to be a sensitive biomarker for urothelial carcinoma and the loss of TM immunoexpression is associated with cell proliferation, increased malignancy and poor prognosis. ${ }^{20-22} \mathrm{TM}$ has also been suggested to be a more sensitive biomarker for TCC than carcinoembryonic antigen because of its lack of immunoreactivity with other tumors and its practical value in separating TCC's from adenocarcinoma of the prostate, colon, bladder, and renal cell carcinomas. ${ }^{22}$ Differentiating between prostate cancer and urothelial carcinomas is important because of therapeutic and prognostic implications.

A protective role for TM has been previously suggested, with decreased expression associated with poor prognosis in patients with metastases in lung, breast and colorectal 


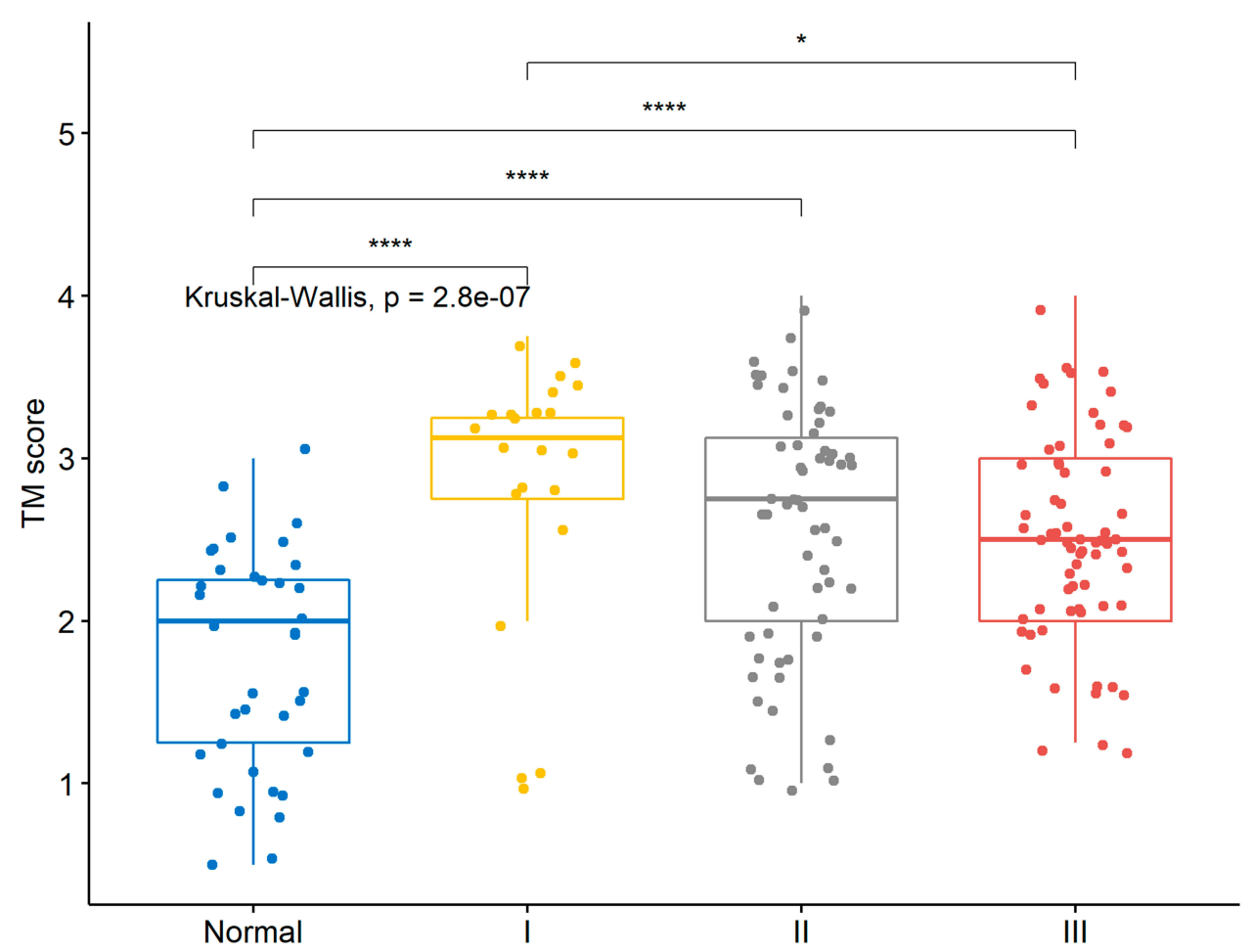

Figure 7 TM immunoexpression scores were significantly lower in normal bladder tissue. TM scores were significantly higher in patients with grade I versus grade III bladder cancer $(* \mathrm{p} \leq 0.05, * * * * \mathrm{p} \leq 0.0001)$.

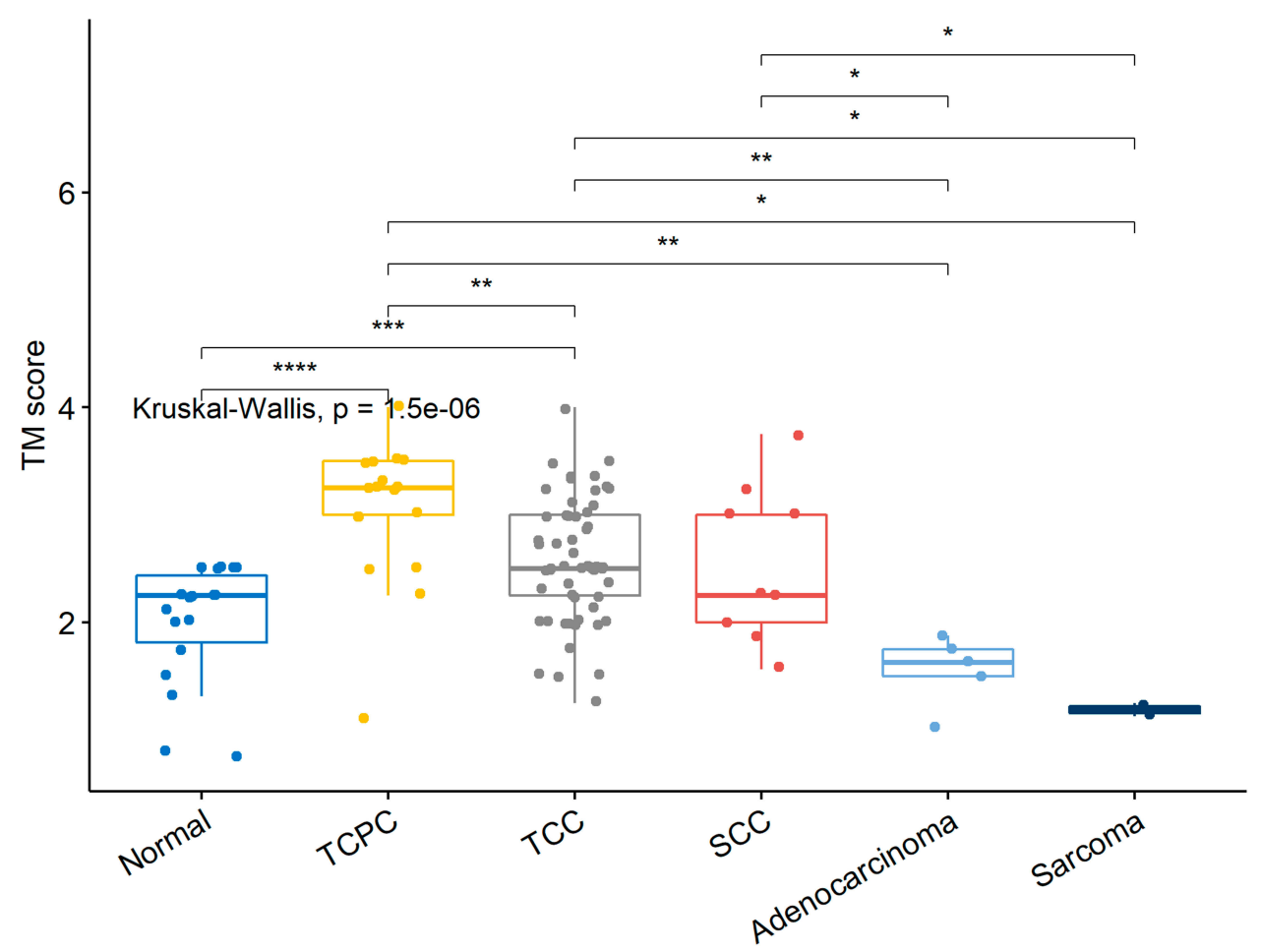

Figure 8 There was a negative relationship between bladder cancer histopathology and TM immunoexpression score. TM immunoexpression scores were stronger in TCPC and TCC with respect to normal tissue, and TCPC, TCC and SCC tissue with respect to adenocarcinoma and sarcoma $(* \mathrm{p} \leq 0.05, * * \mathrm{*} \leq 0.01$, ***p $\leq 0.00 \mathrm{l}$, ****p $\leq$ $0.0001)$. 

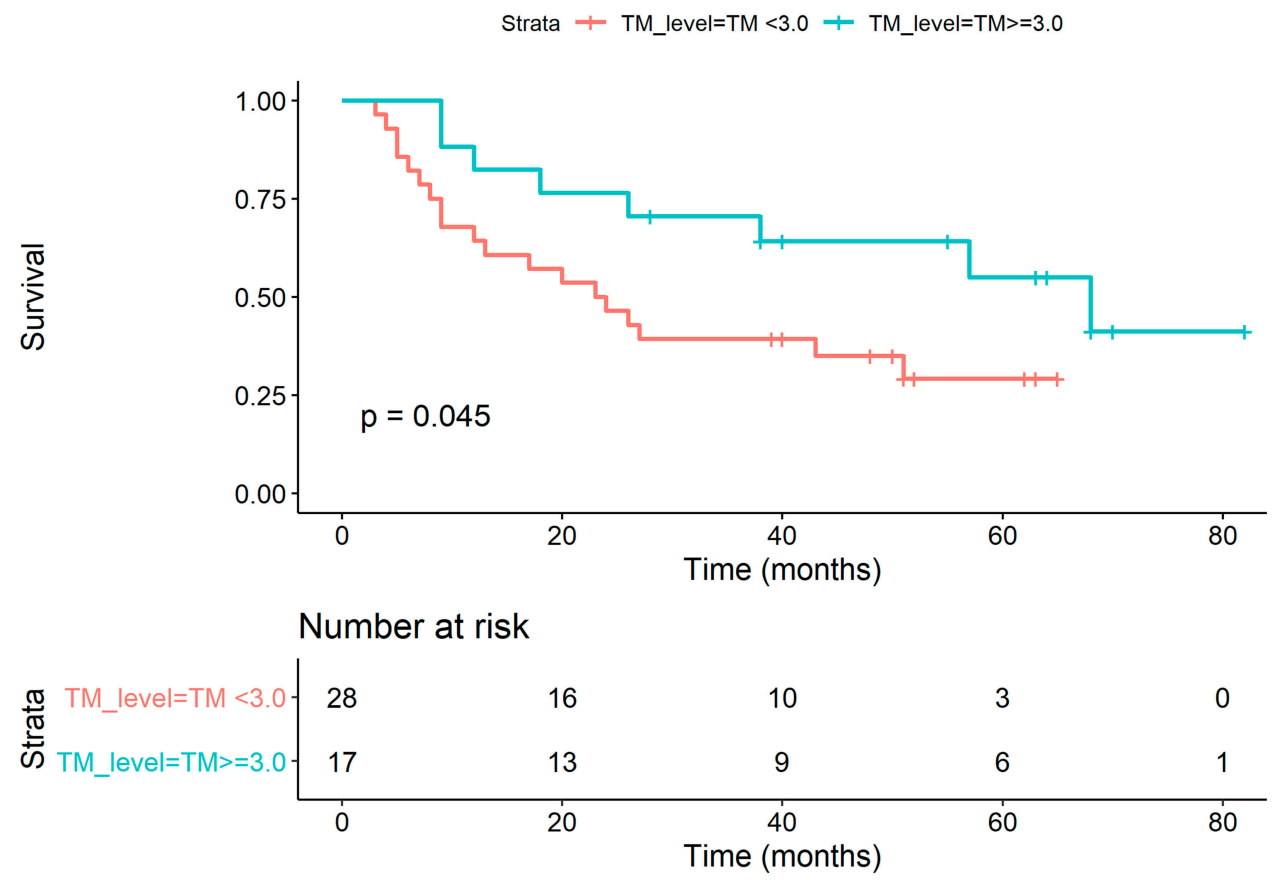

Figure 9 Kaplan-Meier estimates for TM immunoexpression scores versus patient survival (months). Survival data were available for $\mathrm{n}=45$ bladder cancer patients. Applying a TM immunoexpression cut-off score of 3.0 demonstrated that patients with bladder cancer who had a TM immunoexpression score $<3.0$ had lower survival time ( 23.5 months). In contrast, patients with TM immunoexpression scores $\geq 3.0$ had longer survival time (40 months) (log-rank; $p=0.045)$.

cancer. $^{23,24}$ However, the exact molecular mechanism by which protection may be exerted remains unclear. One proposed mechanism hypothesizes that downregulation of TM enhances the tumor cell migratory ability by increasing vimentin expression and decreasing expression of E-cadherin. ${ }^{25,26}$

The prognosis of bladder cancer is dependent on tumor type, grade and stage. Papillary urothelial carcinomas of the bladder usually have a good prognosis. However, SCC and adenocarcinoma of the bladder tend to have a poor prognosis, as these tumors are usually invasive and diagnosed at late stage. In direct contrast, superficial bladder cancer (Ta and T1 tumors) rarely cause death. However, for patients who present with muscle-invasive bladder cancer, the 5-year survival rate is $50 \% .{ }^{27}$ Neoadjuvant chemotherapy has been shown to improve survival however, only in chemosensitive patients. ${ }^{28}$ Generally, prognosis for patients with progressive or recurrent invasive bladder cancer is poor. ${ }^{29}$

Transitional cell papillary carcinoma and TCC of the bladder have a better prognosis than SCC, adenocarcinomas and sarcomas. Patient survival decreases with higher stage and grade at presentation. ${ }^{30-32}$ In this observational pilot study, TM immunoexpression scores were stronger in TCCs (papillary and non-papillary) and SCCs; adenocarcinoma and sarcoma demonstrated weak TM immunoexpression scores. TM immunoexpression scores were higher in grade I and were significantly higher in stage 1 bladder cancer tumors when compared to stage 2-4. However, while this was true, there were several exceptions. TM immunoexpression scores for bladder cancer tissue cores from two patients who were diagnosed with a grade III, stage 3, TCC were compared; one patient had a TM immunoexpression score of 2 and died 9 months after surgery, whereas the second patient, who had a TM immunoexpression score of 4 , is alive and well 82 months after their bladder cancer surgery. As such, these data support previous work that TM immunoexpression may be a positive predictor for patient survival, and the clinical utility of the TM immunoexpression score may be interpreted independently of tumor grade and/or stage. ${ }^{20,33}$

Patients diagnosed with superficial bladder cancer have frequent recurrences throughout their lives. Most of the time, these recurrences are non-invasive and not life threatening. Bladder cancer is, however, the cancer with the highest recurrence rate. Therefore, patients with bladder cancer undergo a lifetime of investigations and treatments to remove their tumors. Risk of progression is based on the number of tumors present in the bladder, whether the tumors are $\geq 3 \mathrm{~cm}$; whether the patient has had bladder cancer before and the grade of the cancer cells. Our data would suggest that TM immunoexpression may provide useful information to the urologist on the tumor 
microenvironment allowing more informed patient management decisions.

Patients presenting with TM immunoexpression scores $\geq 3.0$ had almost 2-fold higher mean survival rates when compared to patients with scores $<3.0$ (40 versus 23.5 months, respectively). Surprisingly, even though the male:female ratio for bladder cancer in our cohort was almost $4: 1$, the female survival rate was lower. It was therefore unsurprising that most of the patients in the survived group were male ( $43 \%$ vs $25 \%)$. Males who present with hematuria are more likely to be referred to urology for investigation. ${ }^{34}$ Furthermore, males are more likely than females to have a complete urological evaluation for their hematuria. ${ }^{34}$ These observations are also borne out by the 1, 5 and 10-year bladder cancer survival rates in males and females. Female bladder cancer patients in England and Wales had almost 20\% lower survival rates at 1 and 5 years, and almost $30 \%$ lower survival rates at 10 years, with respect to males. ${ }^{35}$ This would suggest that female patients are presenting with late stage bladder cancer disease. Unfortunately, female patients who present with hematuria experience more than double the referral wait time for urological investigation, compared to males. $^{36}$

\section{Limitations of the Study}

This was an observational pilot study involving 98 patients. The findings from this study are limited by the patient cohort size and the available survival data. Furthermore, we had no information on patient comorbidities, current medications, and behaviors eg smoking habits or patient treatments. A larger longitudinal study is warranted to demonstrate the significance of our findings.

\section{Conclusion}

In conclusion, our results would suggest that decreased expression of TM in bladder cancer tissue is associated with a poor prognosis; loss of TM expression may indicate increased malignancy and may be a clinically relevant predictor of tumor progression and patient survival.

\section{Abbreviations}

TM, thrombomodulin; IHC, immunohistochemistry; TCPC, transitional cell papillary carcinoma; TCC, transitional cell non-papillary carcinoma; SCC, squamous cell carcinoma; FFPE, formalin-fixed paraffin-embedded; TURBT, transurethral resection of the bladder tumor; TMA, tissue microarrays.

\section{Data Sharing Statement}

The datasets used and/or analyzed during the current study are available from the corresponding author on reasonable request.

\section{Ethics and Consent Statement}

Human tissues cores used in this observational study are de-identified and publicly available. They are thus exempt from the requirement of IRB approval (Exempt Category 4).

\section{Author Contributions}

All authors contributed to data analysis, drafting or revising the article, gave final approval of the version to be published, and agree to be accountable for all aspects of the work.

\section{Funding}

The authors acknowledge the financial support from Invest Northern Ireland (INI) [grant number RD0412515].

\section{Disclosure}

The following authors JW, DGM, CNR, JVL, and MWR work for Randox Laboratories Ltd but hold no shares in the Company. SPF is the Managing Director and owner of Randox Laboratories Ltd. CNR and MWR have a patent thrombomodulin pending. The authors report no other conflicts of interest in this work.

\section{References}

1. Bladder cancer statistics | Cancer Research UK. Available from: https://www.cancerresearchuk.org/health-professional/cancer-statistics /statistics-by-cancer-type/bladder-cancer. Accessed June 20, 2019.

2. Bladder cancer: diagnosis and management $\mid$ guidance | NICE.

3. National Institute for Health and Care Excellence. Bladder cancer: diagnosis and management. NICE Guideline. February, 2015.

4. Pasin E, Josephson DY, Mitra AP, Cote RJ, Stein JP. Superficial bladder cancer: an update on etiology, molecular development, classification, and natural history. Rev Urol. 2008;10(1):31-43.

5. Cohen S, Bryan G, editors. The Pathology ofBladder Cancer. 1st ed. Florida: CRC Press; 1983:1-243.

6. Spruck CH, Ohneseit PF, Gonzalez-Zulueta M, et al. Two molecular pathways to transitional cell carcinoma of the bladder. Cancer Res. 1994;54(3):784-788.

7. Anastasiadis A, de Reijke TM. Best practice in the treatment of nonmuscle invasive bladder cancer. Ther Adv Urol. 2012;4(1):13-32. doi:10.1177/1756287211431976

8. Aldousari S, Kassouf W. Update on the management of non-muscle invasive bladder cancer. Can Urol Assoc J. 2010;4(1):56-64. doi:10.5489/cuaj. 777

9. Svatek RS, Hollenbeck BK, Holmäng S, et al. The economics of bladder cancer: costs and considerations of caring for this disease. Eur Urol. 2014;66(2):253-262. doi:10.1016/j.eururo.2014.01.006 
10. Ploeg M, Aben KKH, Kiemeney LA. The present and future burden of urinary bladder cancer in the world. World J Urol. 2009;27 (3):289-293. doi:10.1007/s00345-009-0383-3

11. Hemani ML, Bennett CL. The excessive cost of early stage bladder cancer care. Cancer. 2010;116(15):3530-3532. doi:10.1002/ cncr. 25227

12. National Institute for Health and Care Excellence. Putting NICE guidance into practice costing statement: suspected cancer implementing the NICE guideline on suspected cancer: recognition and referral. NICE Guideline. 2015; June:1-23.

13. Sievert KD, Amend B, Nagele U, et al. Economic aspects of bladder cancer: what are the benefits and costs? World J Urol. 2009;27 (3):295-300. doi:10.1007/s00345-009-0395-z

14. Abogunrin F, O'Kane HF, Ruddock MW, et al. The impact of biomarkers in multivariate algorithms for bladder cancer diagnosis in patients with hematuria. Cancer. 2012;118(10):2641-2650. doi:10. 1002/cncr.26544

15. Ye F, Wang L, Castillo-Martin M, et al. Biomarkers for bladder cancer management: present and future. Am J Clin Exp Urol. 2014;2(1):1-14.

16. Edge SB, Compton CC. The american joint committee on cancer: the 7th edition of the AJCC cancer staging manual and the future of TNM. Ann Surg Oncol. 2010;17(6):1471-1474. doi:10.1245/s10434010-0985-4

17. Ruddock MW, Reid CN, Reynolds DM, Lamont JV, Fitzgerald SP. Immunodetection of cytoplasmatic membrane-bound thrombomodulin in formalin-fixed paraffin-embedded human tissue microarrays. J Mol Genet Med. 2014;08(04):1-5. doi:10.4172/1747-0862.1000140

18. Van Diest PJ, Van Dam P, Henzen-Logmans SC, et al. A scoring system for immunohistochemical staining: consensus report of the task force for basic research of the EORTC-GCCG. J Clin Pathol. 1997;50(10):801-804. doi:10.1136/jcp.50.10.801

19. R Core Team. R: A Language and Environment for Statistical Computing. 2018.

20. Wu C-T, Chang Y-H, Lin P-Y, Chen W-C, Chen M-F. Thrombomodulin expression regulates tumorigenesis in bladder cancer. BMC Cancer. 2014;14(1):375. doi:10.1186/1471-2407-14-375

21. Ordóñez NG. Thrombomodulin expression in transitional cell carcinoma. Am J Clin Pathol. 1998;110(3):385-390. doi:10.1093/ ajcp/110.3.385

22. Ordonez NG. Value of thrombomodulin immunostaining in the diagnosis of transitional cell carcinoma: a comparative study with carcinoembryonic antigen. Histopathology. 1997;31(6):517-524. doi:10. 1046/j.1365-2559.1997.3040892.x

23. Hanly AM, Redmond M, Winter DC, et al. Thrombomodulin expression in colorectal carcinoma is protective and correlates with survival. $B r J$ Cancer. 2006;94(9):1320-1325. doi:10.1038/sj.bjc. 6603098
24. Kim SJ, Shiba E, Ishii H, et al. Thrombomodulin is a new biological and prognostic marker for breast cancer: an immunohistochemical study. Anticancer Res. 1997;17(3C):2319-2323.

25. Navarro P, Gómez M, Pizarro A, Gamallo C, Quintanilla M, Cano A. A role for the E-cadherin cell-cell adhesion molecule during tumor progression of mouse epidermal carcinogenesis. $J$ Cell Biol. 1991;115(2):517-533. doi:10.1083/jcb.115.2.517

26. Tai CJ, Cheng CW, Su HY, et al. Thrombomodulin mediates the migration of cervical cancer cells through the regulation of epithelial-mesenchymal transition biomarkers. Tumor Biol. 2014;35 (1):47-54. doi:10.1007/s13277-013-1005-7

27. Pagano F, Bassi P, Galetti TP, et al. Results of contemporary radical cystectomy for invasive bladder cancer: a clinicopathological study with an emphasis on the inadequacy of the tumor, nodes and metastases classification. J Urol. 1991;145(1):45-50. doi:10.1016/S00225347(17)38244-7

28. Jayaratna IS, Navai N, Dinney CPN. Risk based neoadjuvant chemotherapy in muscle invasive bladder cancer. Transl Androl Urol. 2015;4(3):273-282. doi:10.3978/j.issn.2223-4683.2015.06.07

29. Park JC, Citrin DE, Agarwal PK, Apolo AB. Multimodal management of muscle-invasive bladder cancer. Curr Probl Cancer. 2014;38 (3):80-108. doi:10.1016/j.currproblcancer.2014.06.001

30. Prognosis and survival for bladder cancer - canadian cancer society. Available from: https://www.cancer.ca/en/cancer-information/cancertype/bladder/prognosis-and-survival/?region=bc. Accessed June 28, 2019.

31. Bladder cancer - genitourinary disorders - MSD manual professional edition. Available from: https://www.msdmanuals.com/en-gb/profes sional/genitourinary-disorders/genitourinary-cancer/bladder-cancer Accessed June 28, 2019

32. PDQ Adult Treatment Editorial Board. Bladder Cancer Treatment $\left(P D Q^{\circledR}\right)$ : Health Professional Version. 2002.

33. Chang YJ, Cheng YW, Lin RK, et al. Thrombomodulin influences the survival of patients with non-metastatic colorectal cancer through epithelial-to-mesenchymal transition (EMT). PLoS One. 2016;11(8): e0160550. doi:10.1371/journal.pone.0160550

34. Osterweil $\mathrm{N}$ Hematuria overlooked as sign of bladder cancer in women.[Updated 2014]. Available from: https://www.medscape. com/viewarticle/825327. Accessed June 20, 2019.

35. Ruddock MW, Reid CN, Lamont JV, Fitzgerald SP. One, 5 and 10 year net survival rates for male and female bladder cancer patients; why are they so different? J Womens Heal Care. 2015;04 (08):1-4. doi:10.4172/2167-0420.1000291

36. Santos F, Dragomir A, Kassouf W, Franco E, Aprikian A. Urologist referral delay and its impact on survival after radical cystectomy for bladder cancer. Curr Oncol. 2015;22(1):e20. doi:10.3747/CO.22. 2052
Research and Reports in Urology

\section{Publish your work in this journal}

Research and Reports in Urology is an international, peer-reviewed, open access journal publishing original research, reports, editorials, reviews and commentaries on all aspects of adult and pediatric urology in the clinic and laboratory including the following topics: Pathology, pathophysiology of urological disease; Investigation and treatment of urological disease; Pharmacology of drugs used for the treatment of urological disease. The manuscript management system is completely online and includes a very quick and fair peer-review system, which is all easy to use. Visit http://www.dovepress.com/ testimonials.php to read real quotes from published authors. 